Supplement of

\title{
A high-altitude balloon platform for determining exchange of carbon dioxide over agricultural landscapes
}

\author{
Angie Bouche et al. \\ Correspondence to: Mark J. Potosnak (mpotosna@depaul.edu)
}

The copyright of individual parts of the supplement might differ from the CC-BY 3.0 licence. 


\section{HYSPLIT}

To assess if there were significant differences in source regions between the two flights, the NOAA Hybrid Single-Particle Lagrangian Integrated Trajectory (HYSPLIT, version 4) model was run for each date. The off-line Windows version was run (http://ready.arl.noaa.gov/HYSPLIT_hytrial.php).

5 Back trajectories for the launch location at Pontiac were run hourly from 18:00 to 21:00 UTC (13:00 - 16:00 CDT). Since the model time frame was 17:00 - 21:00, all back trajectories ended at 17:00. By comparing the first and last back trajectories (18:00 and 21:00, both indicated in red) in each plot, the changes in the source region can be assessed. The trajectories were calculated using an altitude of $1000 \mathrm{~m}$, which was $789 \mathrm{~m}$ above ground level using the the digital elevation model

10 embedded in HYSPLIT. The Eta Data Assimilation System (EDAS from the NWS) archived files (40km resolution) were used as the input meteorology.

Visually assessing the differences in the one-hour back trajectories started at 18:00 and 21:00 (indicated by red triangles closest to the trajectory start) for each of the dates, there are relatively small changes due to changes in wind speed and direction. 
NOAA HYSPLIT MODEL

Backward trajectories ending at 2100 UTC 17 Jul 14

EDAS Meteorological Data

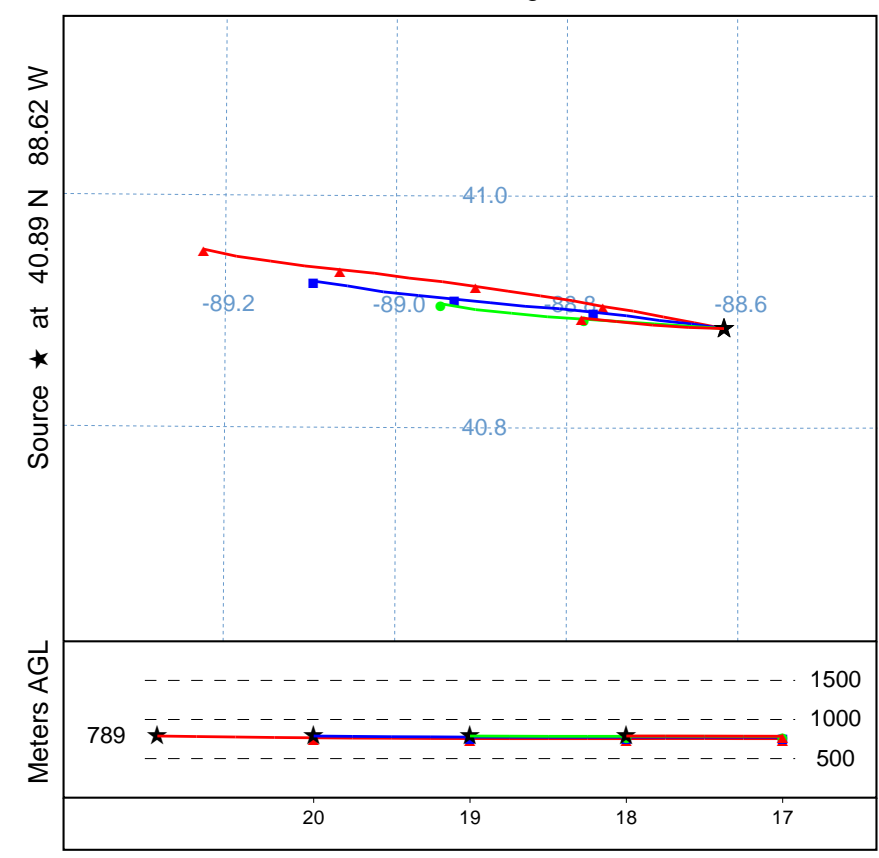

Figure 1. 17 Jul 2014. 
NOAA HYSPLIT MODEL

Backward trajectories ending at 2100 UTC 14 Aug 14

EDAS Meteorological Data

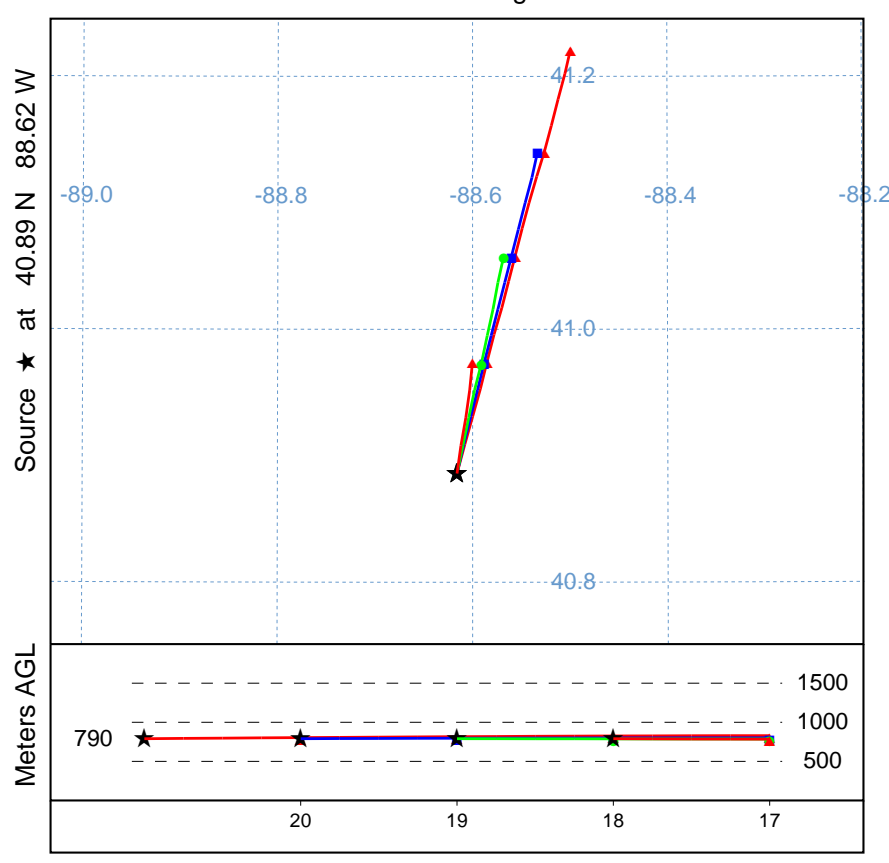

Figure 2. 14 Aug 2014. 
NOAA HYSPLIT MODEL

Backward trajectories ending at 2100 UTC 21 Aug 14

EDAS Meteorological Data

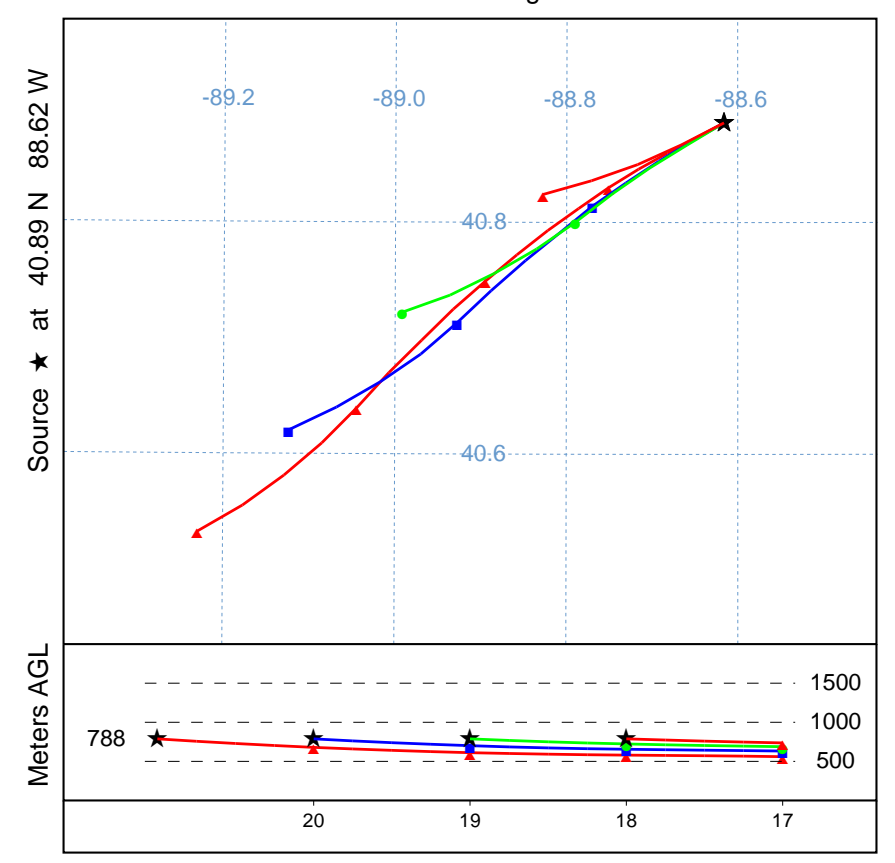

Figure 3. 21 Aug 2014. 
NOAA HYSPLIT MODEL

Backward trajectories ending at 2100 UTC 19 Sep 14

EDAS Meteorological Data

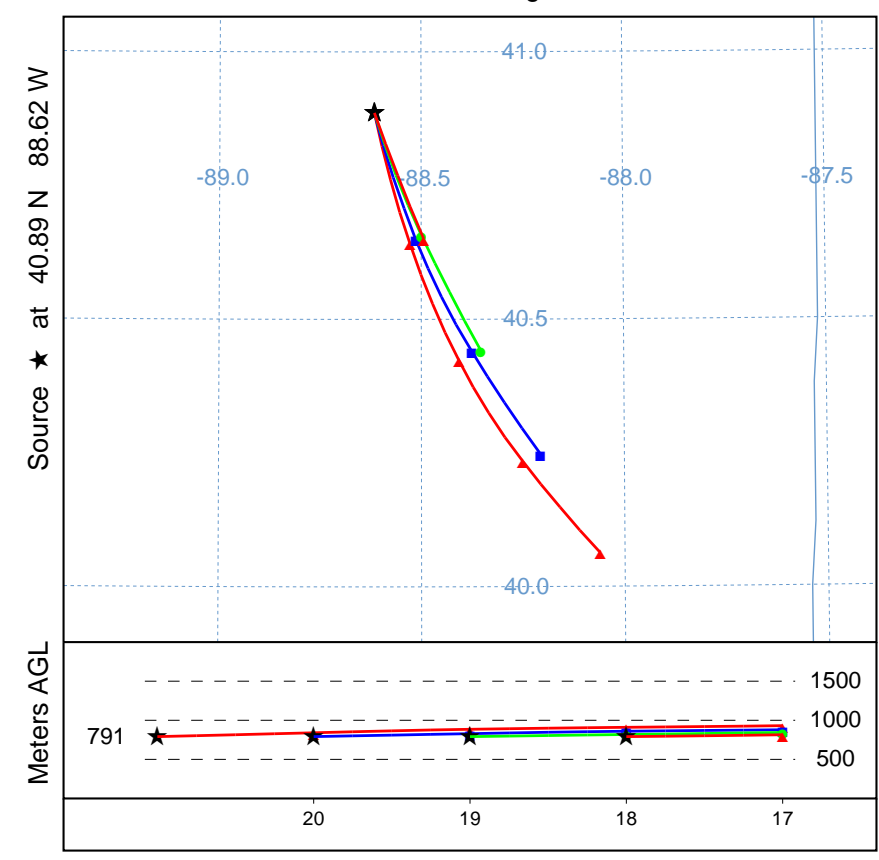

Figure 4. 19 Sep 2014. 
NOAA HYSPLIT MODEL

Backward trajectories ending at 2100 UTC 19 Jun 15

EDAS Meteorological Data

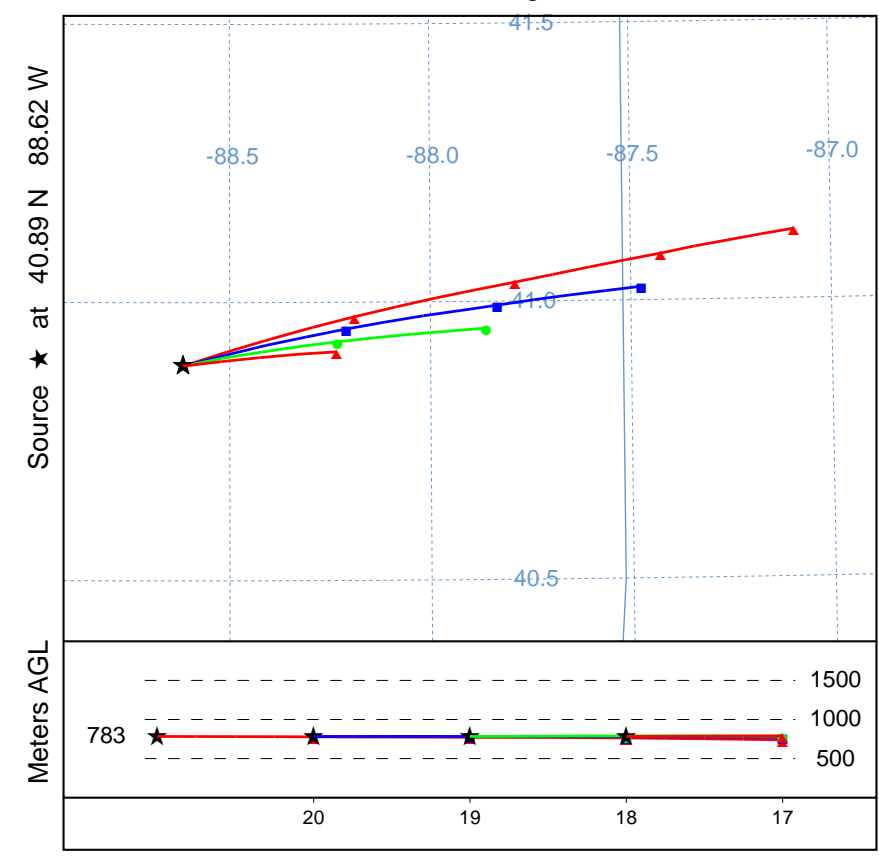

Figure 5. 19 Jun 2015. 
NOAA HYSPLIT MODEL

Backward trajectories ending at 2100 UTC 02 Jul 15

EDAS Meteorological Data

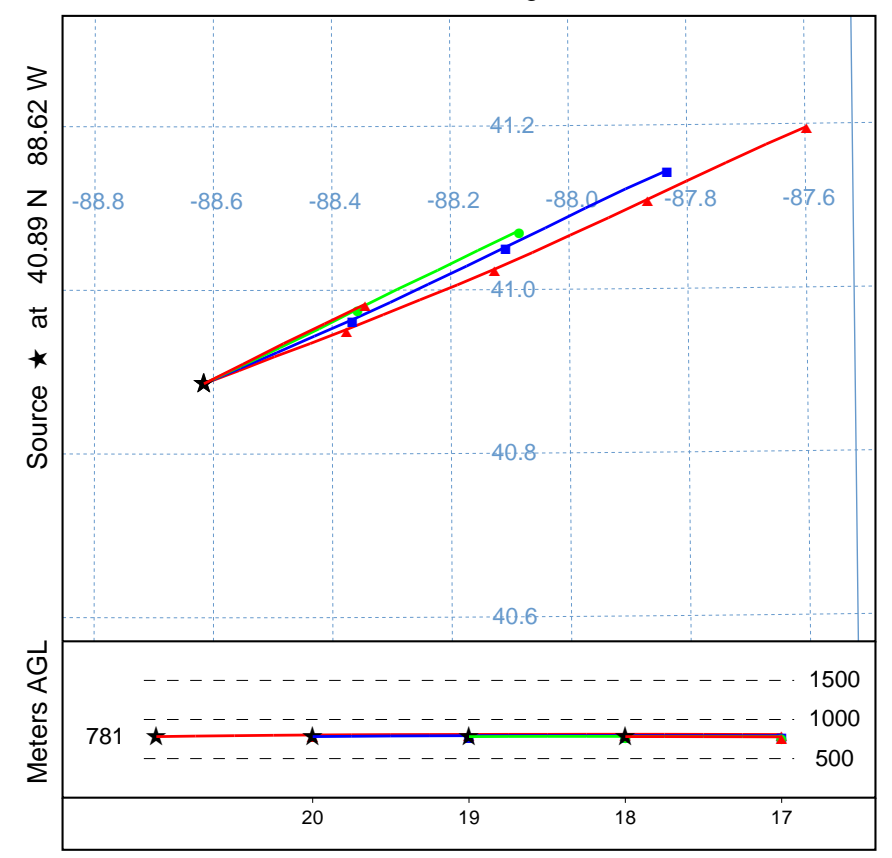

Figure 6. 02 Jul 2015. 
NOAA HYSPLIT MODEL

Backward trajectories ending at 2100 UTC 15 Jul 15 EDAS Meteorological Data

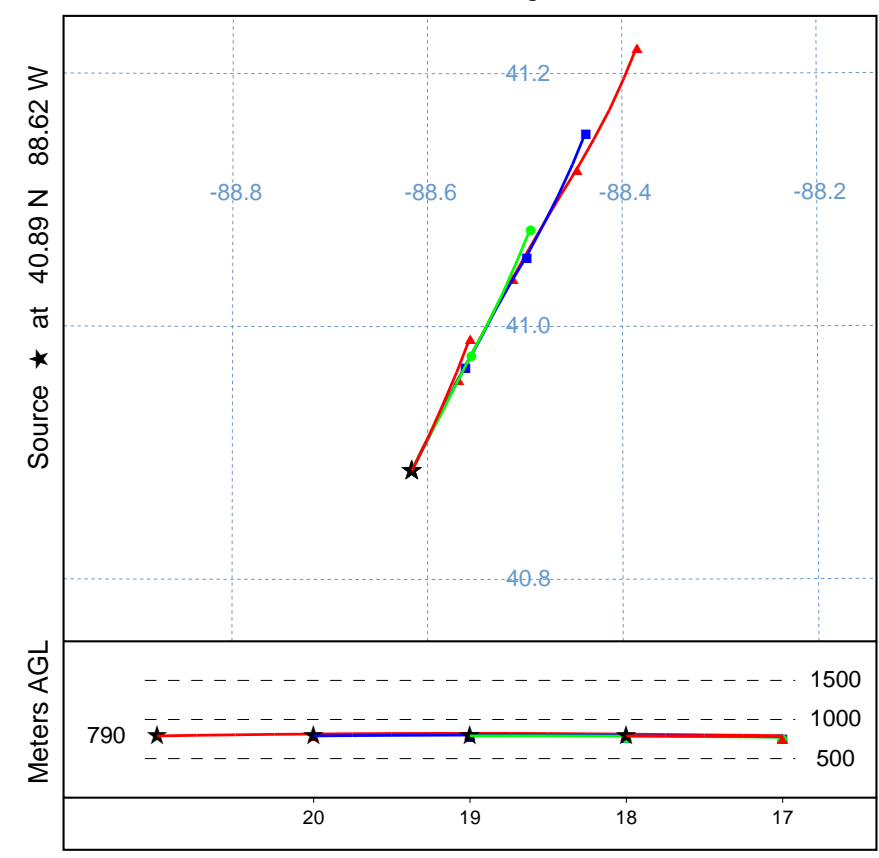

Figure 7. 15 Jul 2015. 
NOAA HYSPLIT MODEL

Backward trajectories ending at 2100 UTC 23 Jul 15

EDAS Meteorological Data

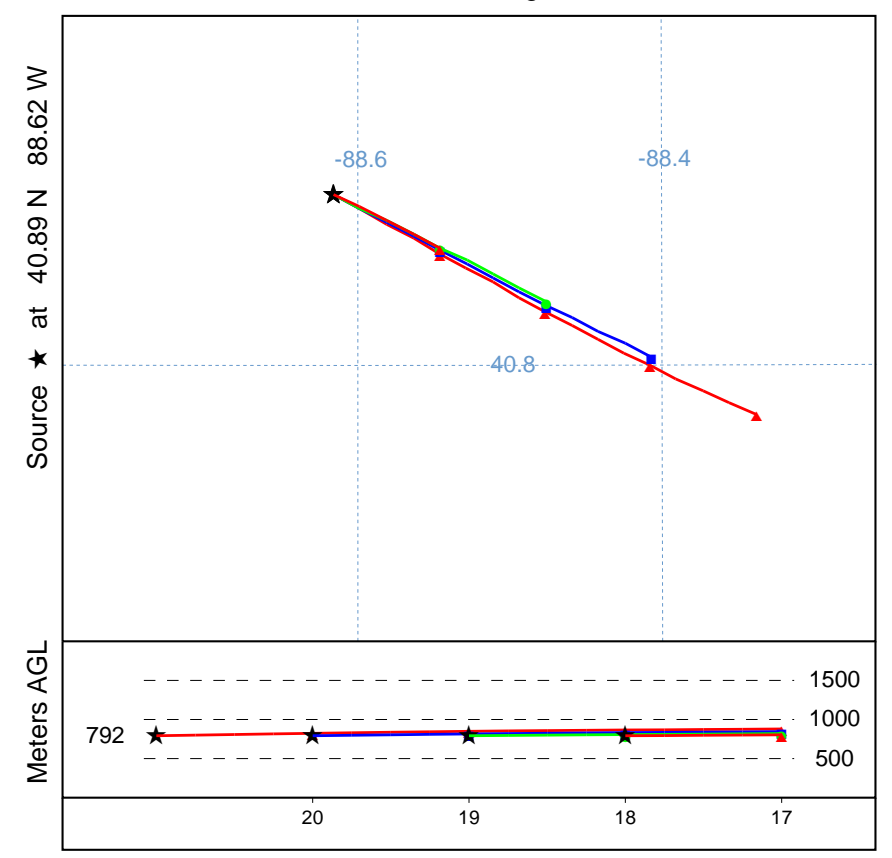

Figure 8. 23 Jul 2015. 
NOAA HYSPLIT MODEL

Backward trajectories ending at 2100 UTC 13 Aug 15

EDAS Meteorological Data

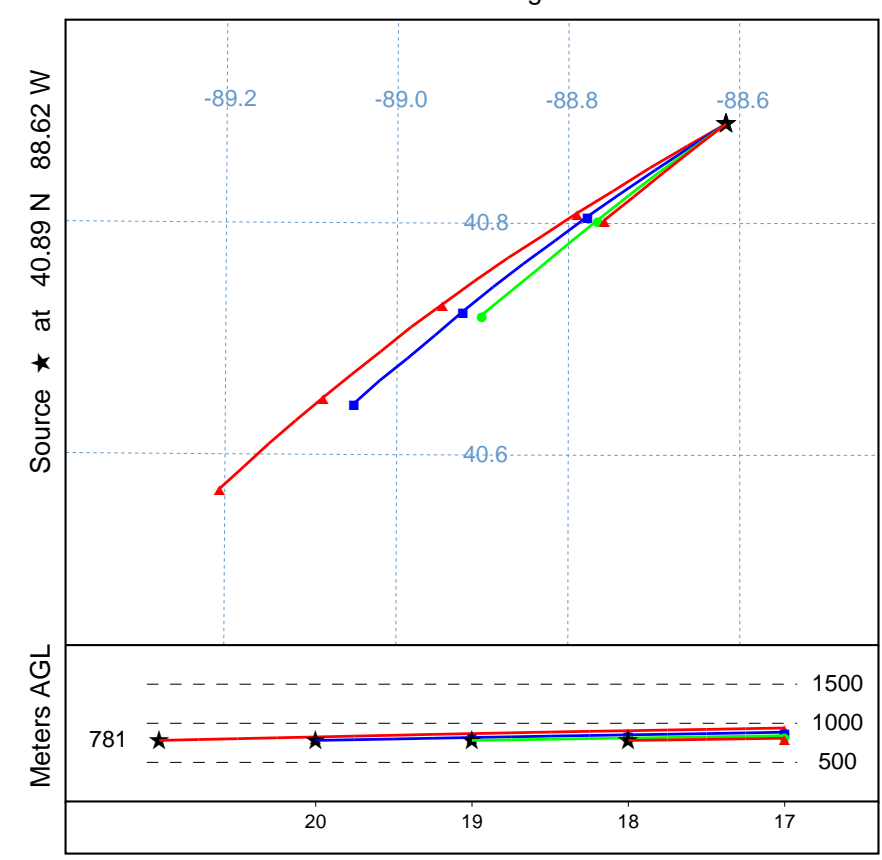

Figure 9. 13 Aug 2015. 
NOAA HYSPLIT MODEL

Backward trajectories ending at 2100 UTC 12 Sep 15

EDAS Meteorological Data

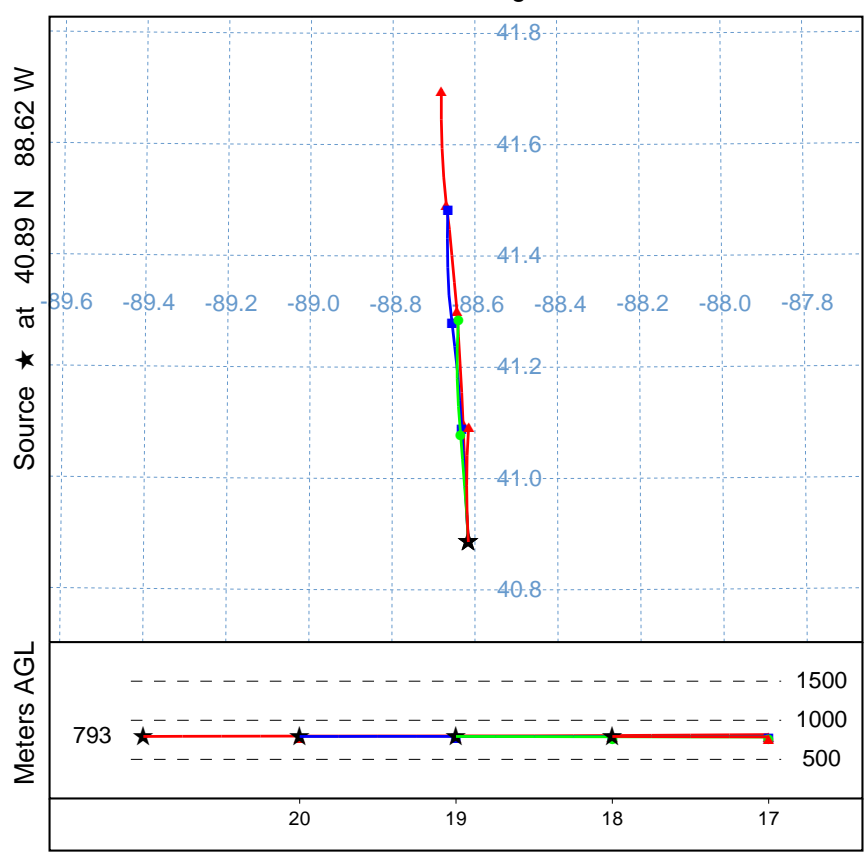

Figure 10. 12 Sep 2015. 